\title{
The Side Force and Yawing Moment in Archaeological Boating Sailing
}

\author{
Yucheng Wang \\ No.268 of Zhang Zhi Dong Road, Wu Chang, Wu Han, Hu Bei, China \\ yuchengw1987@163.com
}

Keywords: side force, yawing moment, heading stability, turbulence model, numerical difference, CFD method.

\begin{abstract}
The ship directional stability is an important index in studying ship maneuverability, but there has not been good method to detect the direction stability of ship. This paper attempts to obtain the side force and yawing moment of fixed symmetrical ship model by using RANS method at steady speed. The side force and yawing moment are introduced in the ship sailing, which affect the directional stability. Finally, the suitable calculation method for lateral force and yaw moment is put forward.
\end{abstract}

\section{Introduction}

Stability of the ship's direction is one of the basic contents of ship maneuverability, and is closely related to the safety of navigation of ships. In recent years, with the promulgation and implementation of ship maneuverability standards and advancement of ship hydrodynamics relevant tests and numerical calculations, researches on ship maneuverability are more and more [1]. Stability of the ship's direction refers to the capacity, which can head back while sailing in a straight line interfered by external disturbances and removed after then. For general ship, the stability of ship direction can be divided into linear motion stability, directional stability and positional stability, depending on the motion parameters. With the development of computing power and computational fluid dynamics, numerical methods are increasingly used in computing ship hydrodynamics, but there are mainly concerned about the unsteady hydrodynamic of underwater or surface vessels. Although it is a long time of study for the ship maneuverability, yet there are few study for course stability in calm water or unstable mechanistic. So far, there has not been quantized research and analysis on the ship's course stability and displacement, draft, depth, speed, ship type etc..[2]

As research about the angle of attack to asymmetric flow field influence deepening, it has been basically clear the asymmetric separated vortex from the object head determines the magnitude of side force and yaw moment, and some fine perturbation at the head may lead to complete inverse of asymmetric aerodynamic forces, which is called the bistable separated vortex structure [3]. However, the uncertainty of this kind of bistable aerodynamic forces will cause trouble on aircraft under high angles of attack flight. In response to this uncertainty, $\mathrm{Hu}$ [4] had forecast the maximum side force coefficient and yawing moment coefficient distribution by using the model test, and studied influence of transition point position. In order to get the side force distribution of different slender body, $\mathrm{Yu}$ [5] simulated the separated vortex flow of a slender body at high angles by solving Reynolds average equations by using unstructured grids and SA turbulence model. In 1977, when Lloyd and Brown studying movement characteristic of spin missiles, found the constant sideways force and yaw moment would cause instability missile sailing. To investigate the effect of side force and yawing moment on spin missile stability, Yuan Tian, Liu Jian xin and Qin [6] had established a nonlinear equation of spin at attack angle. And through the linearization of equation, they gave the effect of side force and yawing moment on dynamic stability by directly using Rolls stability criterion. By comparing calculation results with the results of Lloyd, they verified the correctness of the model and research method, and this model has a better versatility. 


\section{Calculation Model}

\subsection{Governing equations}

By using Fine-Marine software to calculate the high speed vessel model, the governing equations for the continuity equation and Reynolds stress averaged equations are:

$$
\begin{aligned}
& \frac{\partial \rho}{\partial \mathrm{t}}+\frac{\partial}{\partial \mathrm{x}_{i}}\left(\rho \mathrm{u}_{i}\right)=0 \\
& \frac{\partial}{\partial \mathrm{t}}\left(\rho \mathrm{u}_{i}\right)+\frac{\partial}{\partial \mathrm{x}_{j}}\left(\rho \mathrm{u}_{i} \mathrm{u}_{j}\right)=\frac{\partial \mathrm{p}}{\partial \mathrm{x}_{i}}+\frac{\partial}{\partial \mathrm{x}_{j}}\left[\mu\left(\frac{\partial \mathrm{u}_{i}}{\partial \mathrm{x}_{j}}+\frac{\partial \mathrm{u}_{j}}{\partial \mathrm{x}_{i}}-\frac{2}{3} \delta_{i j} \frac{\partial \mathrm{u}_{i}}{\partial \mathrm{x}_{i}}\right)\right]+\frac{\partial}{\partial \mathrm{x}_{j}}\left(-\rho \overline{u_{i}^{\prime} \mathrm{u}_{j}^{\prime}}\right)
\end{aligned}
$$

Where, $\mathrm{u}_{i}$ and $\mathrm{u}_{j}$ are the time average amount of rate, $\mathrm{u}_{i}^{\prime}$ andu $\mathrm{u}_{j}^{\prime}$ are the quantity of velocity fluctuations. And compared to the instantaneous NS equations, given the role of turbulence, an extra entry $\overline{u_{i}^{\prime} u_{j}^{\prime}}$ is Reynolds stresses. The above equation is not closed, in order to achieve a closed equation turbulence model to solve by introducing the Reynolds stress term, achieve fluid control equations can be solved.

\subsection{Turbulence Model}

Compared to other turbulence models, SST turbulence model which is a model based on the model of the evolution of the developed K-Omega model considers the computational precision and convergence in the process of computation [7-10]. It is set in the near-wall region of viscous flow simulation model to simulate the reliability and accuracy advantages model fully developed turbulence model in the region. And equations of the model are as follows:

$$
\begin{aligned}
& \frac{\partial}{\partial \mathrm{t}}(\rho \kappa)+\frac{\partial}{\partial \mathrm{x}_{i}}\left(\rho \kappa \mathrm{u}_{i}\right)=\frac{\partial}{\partial \mathrm{x}_{j}}\left(\Gamma_{\kappa} \frac{\partial \kappa}{\partial \mathrm{x}_{j}}\right)+\mathrm{G}_{\kappa}-\mathrm{Y}_{\kappa} \\
& \frac{\partial}{\partial \mathrm{t}}(\rho \omega)+\frac{\partial}{\partial \mathrm{x}_{i}}\left(\rho \omega \mathrm{u}_{i}\right)=\frac{\partial}{\partial \mathrm{x}_{j}}\left(\Gamma_{\omega} \frac{\partial \omega}{\partial \mathrm{x}_{j}}\right)+\mathrm{G}_{\omega}-\mathrm{Y}_{\omega}+\mathrm{D}_{\omega}
\end{aligned}
$$

In the formula, $\Gamma_{\kappa}$ and $\Gamma_{\omega}$ are the diffusion coefficient, $G_{\kappa}$ and $G_{\omega}$ are the turbulence generation items, $\mathrm{Y}_{\kappa}$ and $\mathrm{Y}_{\omega}$ are the turbulent dissipation terms, $\mathrm{D}_{\omega}$ is the diffusion term.

\subsection{Equations of motion and Calculation Process}

In the process of numerical calculation, the hull movement as a rigid body motion, by using momentum theorem and moment of momentum theorem, we obtained motion equations of the hull six degrees of freedom [11]. Wherein, the movement direction of hull is in the x-axis positive direction, the starboard of hull is in the y-axis positive direction, the anti-direction of gravity is in the $\mathrm{z}$-axis positive direction. In this paper, we consider the impact of hull direct, heave and pitch movement in three directions on resistance at high speed during the voyage.

If you still use the constrained model which does not consider attitude changes of hull to predict the resistance of hull, you will find that there is difference between numerical calculations and experimental values. When the Froude number becomes larger, the gap is more obvious. By using the new calculation method which coupled motion equations of hull and RANS, we timely forecast the force of hull. The hull attitude is adjusting according to the force, until it is in dynamic equilibrium. Thus you can accurately predict the stabilized resistance and attitude of high speed vessel motion.

\subsection{Boundary Conditions}

The calculation domain is $-1.75 \mathrm{Lpp}<\mathrm{x}<2 \mathrm{Lpp},-1.8 \mathrm{Lpp}<\mathrm{y}<1.8 \mathrm{Lpp}, 1.5 \mathrm{Lpp}<\mathrm{z}<0.3 \mathrm{Lpp}$ (Lpp is the length between perpendiculars), where the mid-plane of the ship is located at $y=0$, and ship bottom is at $\mathrm{z}=0$. The stern is at $\mathrm{x}=0$ and the bow at $\mathrm{x}=\mathrm{Lpp}$. At the upper and bottom, the boundary condition prescribed pressure is condition. The downstream is set as 'Far field' boundary. The 'Far field' is given for the calm water calculation on the inlet boundary. The outlet boundary is set as 'Far field' condition. When flow is leaving out of the computational domain, the boundary condition is automatically set as 'Far field' condition in the ISIS code.

\section{Computation Grid}

According to the specific type values of each cross section, by using modeling software to generate three dimensional model of high speed vessel, which is shown in Fig.1. In this paper, in order to compare the calculation results with the corresponding experimental data, we change the scale of real ship in which its reduced scale is 20 with the same scale as ship test model. 
Meshing is one of the most critical parts of the numerical simulation. The quality of the grid directly affects the accuracy of calculation results and convergence time. In this paper, by using the pre-processing Hexpress module in Fine-Marine software, we obtain calculation mesh. The mesh near the surface of hull is suitably refined and projected to the hull surface, thereby body-fitted mesh is forming. At the same time, by splitting the first layer mesh near the hull surface, anisotropic mesh of high quality on boundary layer near the hull surface is generated which is used to accurately capture flow information around the hull. Computational grid is shown in the Fig.1, the overall number of cells is about 305,000 . The mesh elements are almost close to rectangular.

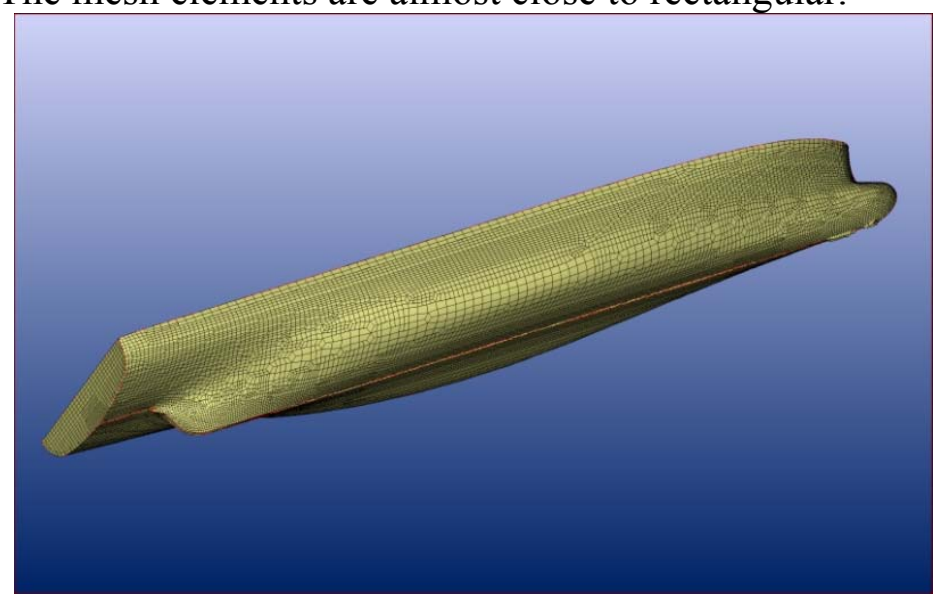

Fig.1 Computational mesh

\section{Numerical Method}

In the numerical calculation process, by using three dimensional incompressible RANS equations as control equations and the center cell finite volume method to discrete momentum equation. Convection term adopt second order upwind difference scheme.

\section{Calculation Results}

In this paper, we calculated the drag coefficient, lateral force coefficient and first swing moment coefficient of ship model at different speed. The calculation results are shown in Fig.2 to Fig.4. The relevant dimensionless coefficients are defined as follows.

$$
C_{x}=\frac{F_{x}}{\frac{1}{2} \rho V^{2} L d}, C_{y}=\frac{F_{y}}{\frac{1}{2} \rho V^{2} L d}, C_{m}=\frac{M_{z}}{\frac{1}{2} \rho V^{2} L^{2} d}
$$

Where, $\mathrm{C}_{x}$ is the drag coefficient, $\mathrm{C}_{y}$ is the transverse force coefficient, $\mathrm{C}_{m}$ is the headed rolling moment coefficient, $\mathrm{F}_{x}$ is the resistance in the direction of $\mathrm{X}$ axis, $\mathrm{F}_{y}$ is the side force in the direction of $\mathrm{Y}$ axis, $\mathrm{M}_{z}$ is the yaw moment for ship, $\mathrm{L}$ is length between perpendiculars, $\mathrm{d}$ for the draft of ship, $\mathrm{V}$ ship speed, $\rho$ for water density. 


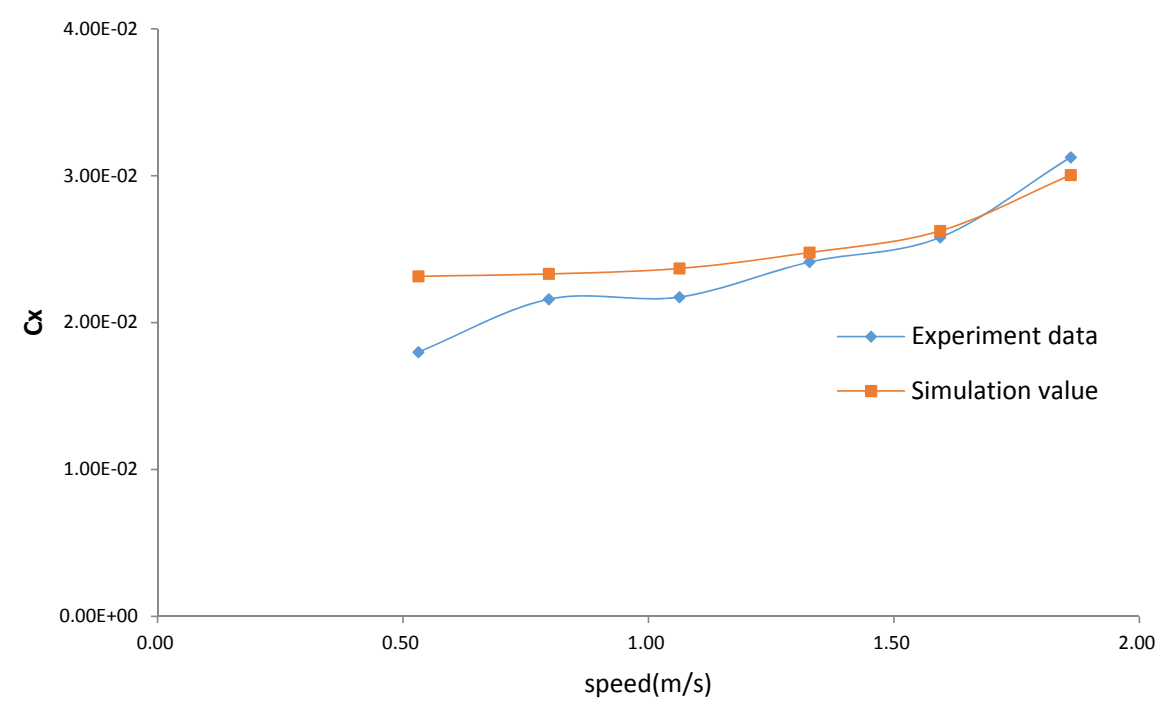

Fig.2 Resistance comparison of model test and numerical calculation

As shown in Fig.2, the difference between the calculation and test of the ship resistance coefficient is smaller and smaller with the increase of the calculation speed. The results show that the data of SSTK $-\omega$ turbulence model is in good agreement with the experimental results. The results show that this method can be used to calculate ship sailing resistance.
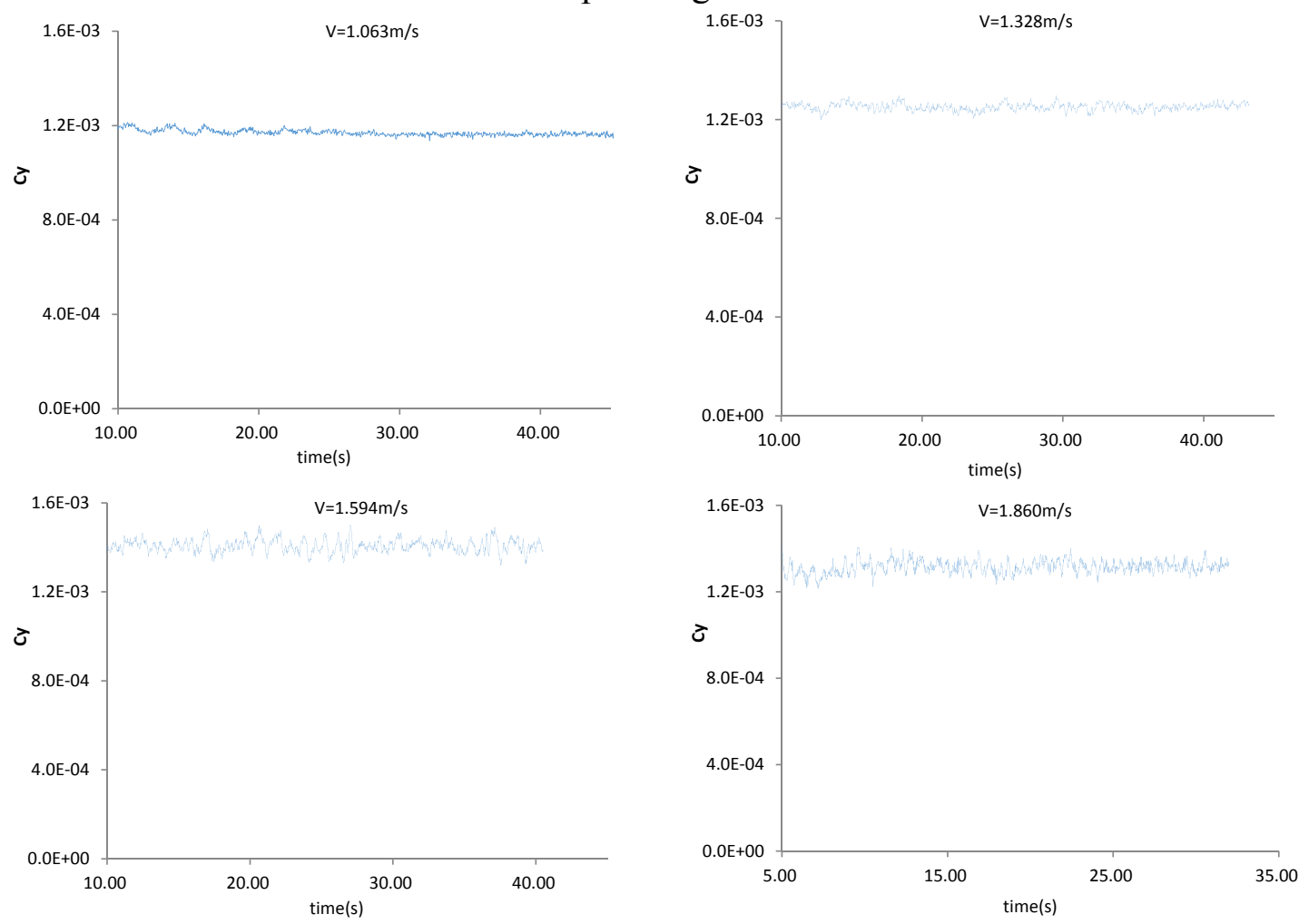

Fig.3 The coefficient of side force

As shown in Fig.3 shows, the lateral force coefficient of hull does not change with time and large amplitude adjustment, when reached to specific computing speed, is stable within a certain range, and changes and fluctuation along with the time. As the increase of the speed, the resistance coefficient will be increased accordingly, and the amplitude of the wave amplitude will be larger. 

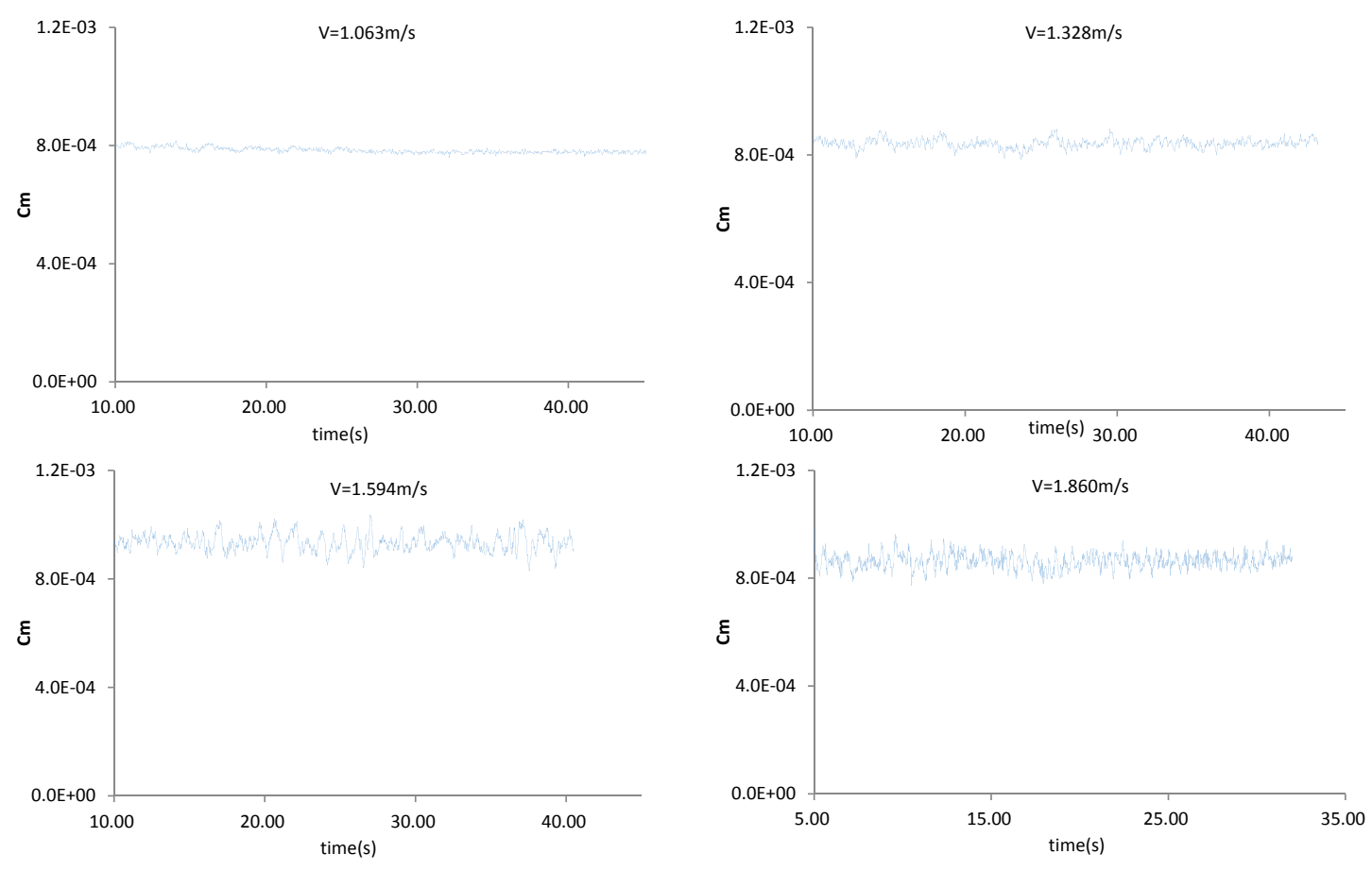

Fig.4 The coefficient of yawing moment

Similar to the change of lateral force coefficient, the stability of swing moment coefficient is stable when the speed is stable, as shown in Fig.4, when the speed of the ship model is $1.063 \mathrm{~m} / \mathrm{s}$. While the speed is increased to $1.860 \mathrm{~m} / \mathrm{s}$, the swing moment coefficient is larger than the numerical value at low speed, and the fluctuation is more intense.
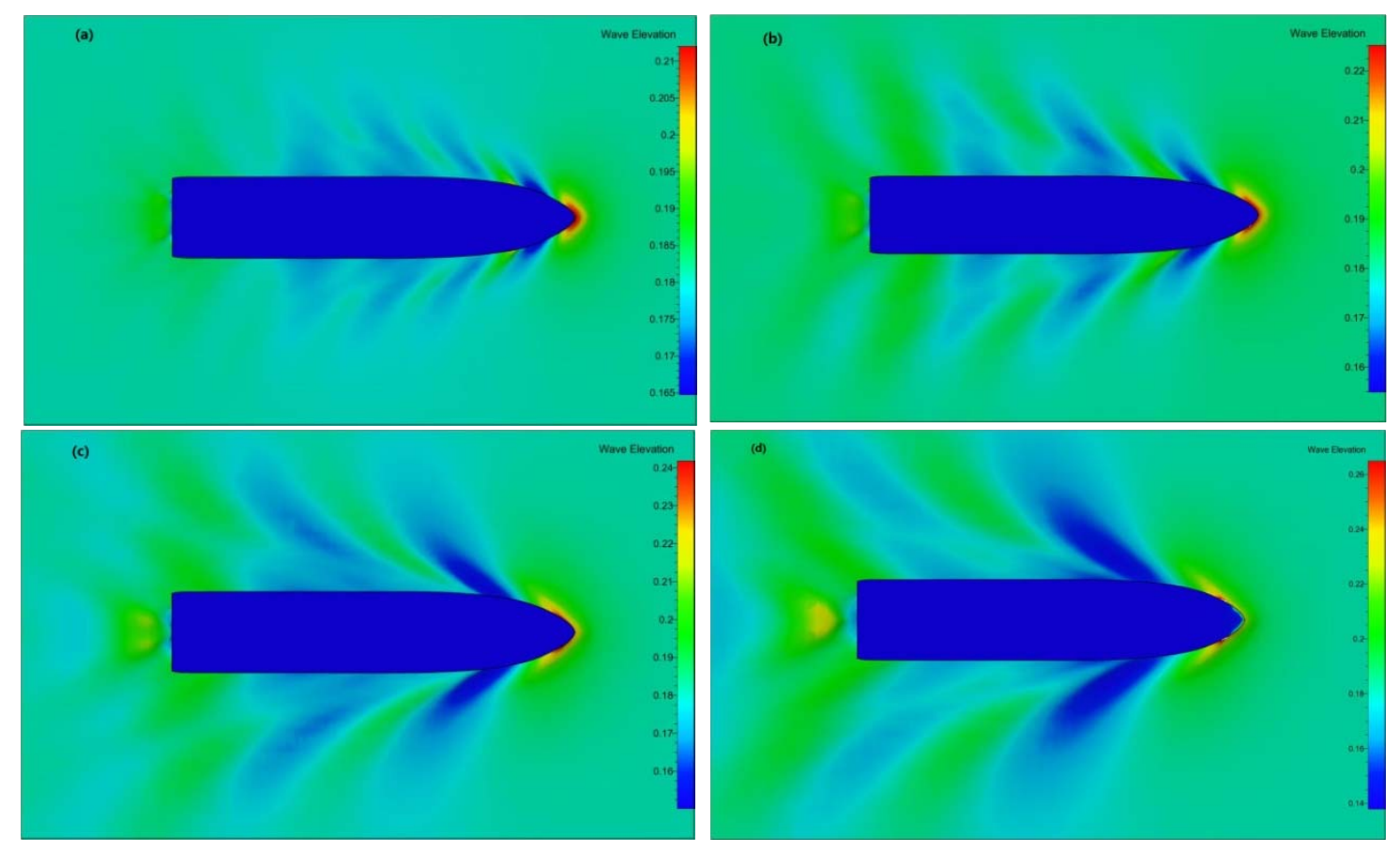

Fig.5 The cloud chart of wave making

The calculation results of free surface under different speed are illustrated in figure 5, in which (a) (d) respectively corresponding speed from $1.063 \mathrm{~m} / \mathrm{s}$ to $1.860 \mathrm{~m} / \mathrm{s}$ wave making contours. Calculated results showed the greater the speed, the higher of wave making generated at the bow and stern, the asymmetric wave surface is also more obvious. 


\section{Conclusion}

In this paper, the commercial computational fluid software Fine-Marine has been used in numerical calculation process. By using STT $\kappa-\omega$ turbulence model to solve the three dimensional incompressible RANS equations, we obtained viscous field near the ship model. We got the wave making contours around the hull, side force and yawing moment coefficients. Thus, the hydrodynamic performance of ships under different speed calculated by numerical simulation method has reached some certain accuracy. It has a certain practical significance for ship type optimization to improve the ship maneuverability.

\section{References}

[1] International Maritime Organization (IMO) Explanatory Notes to the Standards for Ship Maneuverability. MSC/Circ. 1053, 2002.

[2] International Towing Tank Conference(ITTC). The Maneuvring Committee. 2005Final Report and Recommendatimes to the 24th ITTC.

[3] Xing Kaeding Y. Unified Approach to Ship Seakeeping and Maneuvering by a RANSE Method. PhD. Dissertatim, Report No.634, TUHamburg-Harburg, Germany, March. 2006.

[4] Hu Handong, Yang Qide, Zhu MInghong, Deng Xueying. Aircraft Side Force and Yawing Moment Measurement at High Angle of Attack in the Wing Tunnel [J]. Advances in aeronautical Sicence and Engineering.

[5] Yu Kunlong, Ye Zhengyin, Zhang Chenan. Investigation of the Control Technique for Revolution Body with Separated Vortex and Side Force at High Angle of Attack [J]. Aeronautical Computing Technique. Vol.40(2010) No.5,pp:1-4.

[6] Yuan Tianbao, Liu Xinjian, Qin Zizeng. The Effect of Side Force on Stability of Spinning Missiles [J]. Tactical Missile Technology. Vol.2 (2006),pp:54-58.

[7] Deng Rui, Huang Debo, et al. Discussion on factors that affect the flow field CFD calculations catamaran resistance [J] Harbin Engineering University. Vol.32 (2011) No.2, pp: 141-147.

[8] Deng Rui, Huang Debo, Zhou Guangli, et al Preliminary Numerical Investigation of Effect of Interceptor on Ship Resistance [J] Applied Mechanics and Materials.Vol.97-98(2011), pp:10851090.

[9] JMJ Journée. Experiments and Calculations on 4 Wigley Hull Forms in Head Waves [R]. Report 0909, May 1992, Delft University of Technology, Ship Hydromechanics Laboratory, Mekelweg 2, 2628 CD Delft, The Netherlands.

[10] Menter, FR. Influence of free stream values on turbulence model predictions [J].AIAA Journal, Vol.30(1992), No.6.

[11] Menter, F..R. Zonal two-equation turbulence models for aerodynamic flows. AIAA paper (1993)No.93-2906. 